\title{
Correlation between symptoms of the irritable bowel syndrome and the response to the food extract skin prick test
}

R.L.S. Soares,

H.N. Figueiredo,

C.P. Maneschy,

V.R.S. Rocha and

J.M. Santos
Grupo de Estudo de Doenças Intestinais, Departamento de Medicina Clínica, Faculdade de Medicina, Universidade Federal Fluminense, Niterói, RJ, Brasil

\section{Correspondence}

R.L.S. Soares

Departamento de Medicina Clínica

Faculdade de Medicina, CCM, UFF

24030-210 Niterói, RJ

Brasil

E-mail: rsalerno@openlink.com.br

Presented at the XVIII Annual Meeting of the Federação de Sociedades de Biologia Experimental, Curitiba, PR, Brazil, August 27-30, 2003.

Research supported by CNPq (PIBIC) and Fundação Universitária Euclides da Cunha (FEC, UFF).

\begin{abstract}
The relationship between the irritable bowel syndrome (IBS) and food intolerance is not clear. We studied the cutaneous response to food antigens in 43 volunteers who were students and employees of the Faculty of Medicine of Universidade Federal Fluminense. Subjects were divided into 3 groups after evaluation for Roma II criteria for functional disease of the gastrointestinal tract: group I, 14 volunteers with IBS; group II, 15 volunteers with functional dyspepsia; group III, 14 volunteers without habitual gastrointestinal symptoms. The subjects were submitted to the skin prick test with 9 food antigen extracts, for a total of 387 skin tests ( 9 per volunteer). Of the 126 tests applied to group I, 24 (19.4\%) were positive (a 3-mm wider papule than the negative control) and of the 135 tests applied to group II, 3 (2.3\%) were positive. Of the 126 tests applied to group III, $6(4 \%)$ were positive. The number of positive responses obtained in group I (IBS) differed significantly from the other 2 groups $(\mathrm{P}<0.01)$. None of the volunteers with IBS reported intolerance to any isolated food. The higher reactivity to food antigens in group I compared to groups II and III suggests that intestinal permeability may be increased in patients with IBS.
\end{abstract}

Over the last decade, the study of functional diseases of the gastrointestinal tract (FGD), especially irritable bowel syndrome (IBS), has been gaining importance in the field of gastroenterology (1-3). Unlike most of the diseases of the digestive tract which can be diagnosed using biological markers $(1,4)$, FGD can only be diagnosed using clinical approaches, presuming the absence of structural or biochemical dysfunction in the generation of the symptoms (5-9).
Key words

- Irritable bowel syndrome

- Food intolerance

- Skin prick test
IBS is one of the most common diseases in clinical practice. Symptoms of IBS (episodes of abdominal pain associated with alterations in intestinal habits occurring more than six times a year), are observed in $10 \%$ of the general population. The prevalence estimates vary from 9 to $22 \%$ depending on the population studied $(2,3,6)$.

The mechanisms involved in the pathophysiological alterations found in FGD seem to be multiple and are still uncertain. A uni- 
fying hypothesis for the generation of these symptoms would be the phenomenon of visceral hypersensitivity identified in most of the patients with IBS (7).

The phenomenon of visceral hypersensitivity may be related to an increased response of the neuroimmune circuits in the nervous system or gastrointestinal tract to external stimuli (for example environmental or psychosocial stimuli) or internal ones (tissue irritation, inflammation, infection) $(4,5$, 7). This increased response may result in abnormalities of digestive motility, inducing symptoms compatible with the clinical picture of IBS. In synthesis, an abnormal neuroimmune interaction (genetic and psychosocial factors, food intolerance, bacterial microflora) may contribute to the phenomenon of visceral hypersensitivity frequently observed in the patients with IBS (1,4,5,6-9).

Clinically, the frequency of IBS is higher among females and is associated with psychological stress, food intolerance (adverse reaction to a specific food or ingredient that is not immune mediated or associated with psychological phenomena), intestinal infections, and even previous abdominal surgeries $(1,2,6,8,10)$. The pathogenesis of food intolerance, frequently associated with IBS symptoms, has been little studied.

The aim of the present study was to correlate symptoms of IBS with the response to food antigens using the skin prick test. We studied the response to food antigens by the prick test in 43 volunteers, 27 women and 16 men, during the period from August 2002 to January 2003. The subjects were selected from 105 volunteers, students and employees of the Faculty of Medicine, Universidade Federal Fluminense, Niterói, RJ, Brazil, recruited through a poster affixed on the door of the group of study of intestinal diseases (outpatient unit), Hospital Universitário Antonio Pedro.

The inclusion criteria were age above 18 years and volunteers from the general population. This approach was used in order to obtain a population sample that would not consist of ambulatory or hospital patients, but that would represent the population in general. The exclusion criterium was the diagnostic suspicion of organic disease of the gastrointestinal tract.

The study consisted of 2 stages taking place over 2 consecutive weeks.

Stage I. Clinical evaluation of the volunteers, including the application of Roma II criteria (1) for the determination of IBS (pain or abdominal discomfort accompanied by 2 or 3 symptoms, such as relief with defecation and/or with alterations in the frequency of evacuations or in the shape of the feces for at least 12 weeks, which need not be consecutive, in the preceding 12 months; in the absence of organic disease of the gastrointestinal tract and functional dyspepsia, persistent or recurrent symptoms, such as pain or discomfort in the upper abdomen at least 12 weeks earlier, not necessarily serial, during the preceding 12 months).

Stage II. Application of skin tests using food antigens by the prick test.

Of the 105 volunteers, 30 were excluded because of a diagnostic suspicion of organic disease of the gastrointestinal tract and were scheduled for later outpatient consultation, 10 did not agree to be submitted to the skin tests, and 22 did not return the following week for the scheduled skin tests.

The 43 selected volunteers were divided into 3 groups after clinical evaluation and the application of Roma II's approaches for IBS and functional dyspepsia. Group I consisted of 14 volunteers with a diagnosis of IBS (10 women and 4 men; mean age 25 years), group II consisted of 15 volunteers with a diagnosis of functional dyspepsia ( 9 women and 6 men; mean age 26 years), and group III consisted of 14 volunteers without habitual previous symptoms of gastrointestinal tract disease ( 8 women and 6 men; mean age 28 years).

The three groups were submitted to the skin tests with 9 food extracts (egg white, 
egg yolk, peanut, chestnut, wheat flour, cow milk, soy, crustaceans, and chocolate).

We used the commercially available allergenic reagents (M. Queiroz Laboratory, Rio de Janeiro, RJ, Brazil) in 50\% glycerin diluent. The antigens were applied on the volar surface of the forearm in droplets ( 25 $\mathrm{ml}$ of testing reagent) by the technique of percutaneous puncture (11) using identical needles and were discarded after each test to avoid cross-reactions due to allergen absorption at multiple skin sites.

Twenty minutes after antigen application, the skin tests were read by measuring the diameter of the papule in millimeters. A papule $3 \mathrm{~mm}$ wider than the negative control was considered to be a positive response, whereas any smaller value was considered to be negative (11).

The study was approved by the Health Research and Post-Graduation Adviser Committee, Research and Pos-Graduation Department, UFF.

A total of 387 skin tests were applied to the 3 groups ( 9 for each volunteer). In group I, IBS, 24 positive responses were obtained, corresponding to $19.4 \%$ of the 126 tests performed. In group II, functional dyspepsia, 3 positive responses were obtained, corresponding to $2.3 \%$ of the 135 tests performed. In group III, 6 positive responses were obtained, corresponding to $4 \%$ of the 126 tests performed (Table 1 ). The positive responses were not concentrated in 1 or 2 individuals in each group but were dispersed throughout the group.

Ten volunteers with IBS presented a positive response to one or more food antigens but the positive response was not specifically associated with IBS crises in any of them.

The number of positive response was not significantly different between groups II and III. In group I, IBS, the number of positive responses differed significantly from the val-
Table 1. Responses of patients with irritable bowel syndrome to the food antigen prick test.

\begin{tabular}{lccc}
\hline & $\begin{array}{c}\text { Irritable bowel } \\
\text { syndrome }\end{array}$ & $\begin{array}{c}\text { Functional } \\
\text { dyspepsia }\end{array}$ & $\begin{array}{c}\text { Without intestinal } \\
\text { symptoms }\end{array}$ \\
\hline No. of subjects & 14 & 15 & 14 \\
No. of tests & 126 & 135 & 126 \\
No. of positive responses & $24^{*}$ & 3 & 6 \\
$\%$ positive responses & $19.4 \% *$ & $2.3 \%$ & $4.0 \%$ \\
\hline
\end{tabular}

A papule $3 \mathrm{~mm}$ larger than the negative control was considered to be a positive response. The positive antigens (number) for irritable bowel syndrome were: cow milk (4), egg yolk (4), chocolate (3), wheat flour (3), nuts (3), soy protein (2), egg albumin (1), peanuts (1), and crustaceans (1); for functional dyspepsia were: cow milk (1), chocolate (1), and wheat flour (1), and for patients without intestinal symptoms were: wheat flour (1), nuts (1), egg albumin (1), chocolate (1), and peanuts (1). ${ }^{*} P<$ 0.05 compared to the other groups (Student $t$-test).

ues obtained in the other 2 groups $(\mathrm{P}<0.01$, Student $t$-test).

The present results agree with those reported by others. The association between IBS and sensitivity to multiple foods has also been reported in most of the few available studies in the literature $(10,12)$. None of the volunteers with IBS reported intolerance to any isolated food $(10,13,14)$.

This finding suggests that patients with IBS symptoms have difficulties with foods in general. It is very probable that IBS causes food intolerance and not the opposite (1519). Despite the small number of cases studied, the higher reactivity to food antigens in group I compared to groups II and III adds a new information and suggests the presence of a possible alteration in intestinal epithelial function (20).

The association between phenomena of food hypersensitivity and IBS symptoms is still open to question. Future clinical investigations on larger samples using tests of intestinal permeability will be useful for a better understanding of the results obtained here. 


\section{References}

1. Drossman DA, Corazziari E, Talley NJ, Thompson WG \& Whitehead WE (2000). Rome - The Functional Gastrointestinal Disorders. 2nd edn. Allen Press, McLean, VA, USA.

2. Locke GR (1996). The epidemiology of functional gastrointestinal disorders in North America. Gastroenterology Clinics of North America, 25: 1-19

3. Talley NJ, Zinsmeister HR \& Mellton LJ (1995). Irritable bowel syndrome in a community: symptom subgroups, risk factors, and health care utilization. American Journal of Epidemiology, 142: 7683.

4. Mayer EA \& Collins SM (2002). Evolving pathophysiologic models of functional gastrointestinal disorders. Gastroenterology, 122: 20322048.

5. Downing JE \& Miyan JÁ (2000). Neural immunoregulation: emerging roles for nerves in immune homeostasis and disease. Immunology Today, 21: 281-289.

6. Jones R \& Lydeard S (1992). Irritable bowel syndrome in the general population. British Medical Journal, 304: 87-90.

7. Cooke HJ (2000). Neurotransmitters in neuronal reflexes regulating intestinal secretion. Annals of the New York Academy of Sciences, 915: 77-80.

8. Toner BB \& Akman D (2000). Gender role and irritable bowel syndrome: literature review and hypothesis. American Journal of Gastroenterology, 95: 11-16.

9. Barbara G, De Giorgio R, Stanghellini V, Cremon C \& Corinaldesi R (2002). A role for inflammation in irritable bowel syndrome? Gut, 51 (Suppl I): I-141-I-144.

10. Crowe SE \& Perdue MH (1992). Gastrointestinal food hypersensitivity: basic mechanisms of pathophysiology. Gastroenterology, 103: 1075-1095.
11. Bernstein L \& Storms WW (1995). Practice parameters for allergic testing. Annals of Allergy, Asthma, and Immunology, 75: 543-625.

12. Brandtzaeg $P$ (2002). Current understanding of gastrointestinal immunoregulation and its relation to food allergy. Annals of the New York Academy of Sciences, 964: 13-45.

13. Ahmed T \& Fuchs GJ (1997). Gastrointestinal allergy to food: a review. Journal of Diarrhoeal Diseases Research, 15: 211-223.

14. Nice AM, Frankum B \& Talley NJ (1998). Are adverse food reactions linked to irritable bowel syndrome? American Journal of Gastroenterology, 93: 2184-2190.

15. Jenkins $L$ \& Zwetchkenbaum $R$ (1988). The irritable bowel syndrome and food hypersensitivity. Annals of Allergy, 61: 47-49.

16. Locke GR, Zeismeister AR, Talley NJ, Kett SL \& Melton LJ (2000). Risk factors for irritable bowel syndrome: role of analgesics and food sensitivities. American Journal of Gastroenterology, 95: 157165.

17. Petitpierre M, Gumowski P \& Girard JP (1985). Irritable bowel syndrome and hypersensitivity to food. Annals of Allergy, 54: 538540.

18. Soares RLS, Figueiredo HN, Dick MFS, Silva Filho NF, Lima LFQ \& Santos JM (2001). A síndrome do intestino irritável associada à intolerância alimentar - criação de um perfil clínico-epidemiológico regional. Avaliação em duas regiões geográficas brasileiras Sudeste e Amazônia. Mundo \& Vida, 2: 20-32.

19. Jones VA, Shorthouse M, McLaughlan P, Workman E \& Hunter JO (1982). Food intolerance: a major factor in the pathogenesis of irritable bowel syndrome. Lancet, ii: 1115-1117.

20. Unno N \& Fink MP (1998). Intestinal epithelial hyperpermeativity mechanisms - relevance to disease. Gastroenterology Clinics of North America, 27: 289-307. 